\title{
Searching for boosted dark matter mediated by a new gauge boson
}

\author{
Wonsub Cho®, ${ }^{*}$ Ki-Young Choi, ${ }^{\dagger}$ and Seong Moon Yoo ${ }^{\ddagger}$ \\ Department of Physics, Sungkyunkwan University, \\ 2066, Seobu-ro, Jangan-gu, Suwon-si, Gyeong Gi-do, 16419 Korea
}

(Received 13 July 2020; accepted 16 October 2020; published 12 November 2020)

\begin{abstract}
We study the possibility to directly detect the boosted dark matter generated from the scatterings with high energetic cosmic particles, such as protons and electrons. As a concrete example, we consider the sub-GeV dark matter mediated by a $\mathrm{U}(1)_{D}$ gauge boson, which has a mixing with a $U(1)_{Y}$ gauge boson in the Standard Model. The enhanced kinetic energy of the light dark matter from the collision with the cosmic rays can recoil the target nucleus and electron in the underground direct detection experiments, transferring enough energy to them to be detectable. We show the impact of boosted dark matter with existing direct detection experiments as well as collider and beam-dump experiments.
\end{abstract}

DOI: 10.1103/PhysRevD.102.095010

\section{INTRODUCTION}

The nature of dark matter (DM) is one of the unsolved problems in the astro-particle physics that spans from the small scales of the Galaxy to the large scales of the Universe [1]. The underground direct-detection experiment is one of the ways to search for the nongravitational nature of DM, and the sensitivity of the elastic scattering cross section with a nucleon now goes down to $\sigma_{\chi p} \gtrsim 4.1 \times$ $10^{-47} \mathrm{~cm}^{2}$ at $30 \mathrm{GeV}$ of DM mass [2]. The constraints on the scattering cross section of DM with an electron is $\sigma_{\chi e} \gtrsim 3 \times 10^{-38} \mathrm{~cm}^{2}$ at $100 \mathrm{MeV}$ [3-5].

In these studies of the DM direct detection, the DMs are assumed to be nonrelativistic with a Mawell-Boltzmann distribution around the Milky Way galaxy, with a speed around $10^{-3} c$, with the speed of light $c$. However, recently, it was noticed that the elastic scattering of DMs in the Milky Way with a cosmic ray can change the cosmic ray spectra [6] and also boost DM [7-9]. The boosted DM (BDM) can transfer large momentum to the target and make the recoil energy above the detector threshold even with the light DM. This was used to search for dark matter in simple models [10-14].

In this paper, we apply this novel method to the light DM mediated by a new $\mathrm{U}(1)_{D}$ gauge boson, which has a mixing with $\mathrm{U}(1)_{Y}$ in the Standard Model (SM) [15-17], which is

\footnotetext{
*sub526@skku.edu

†kiyoungchoi@skku.edu

castledoor@skku.edu
}

Published by the American Physical Society under the terms of the Creative Commons Attribution 4.0 International license. Further distribution of this work must maintain attribution to the author(s) and the published article's title, journal citation, and DOI. Funded by SCOAP ${ }^{3}$. one of the simplest extensions of the SM. In this model, the mixing connects the visible and hidden sectors through the mediation of the gauge bosons and opens the portal to the DM in the hidden sector. Here, the DMs can interact with both nuclei and electrons, and therefore, it is necessary to consider both scatterings with nuclei and electrons in the BDM generation, as well as in the direct detection. This gives different behavior and constraints compared to the previous analysis, assuming a single kind of interaction. In this study, we give the realization of the upscattered DM by cosmic rays of a vector mediation [10] and complement the existing constraints on this model [18-27].

In Sec. II, we introduce the model we consider, and in Sec. III, we summarize the generation of BDM and attenuation. In Sec. IV, we show the results with constraints from BDM and conclude in Sec. VI.

\section{MODEL}

We consider a model of Dirac fermion dark matter with a dark gauge symmetry $\mathrm{U}(1)_{D}$, which mediates the interaction between dark and SM sectors through mixing with $U(1)_{Y}$ in the Standard Model [21,28,29]. The Lagrangian is given by

$\mathcal{L}_{Z_{d}}=-\frac{1}{4} \hat{Z}_{d \mu \nu} \hat{Z}_{d}^{\mu \nu}+\frac{\sin \varepsilon}{2} \hat{B}_{\mu \nu} \hat{Z}_{d}^{\mu \nu}+\frac{1}{2}\left(m_{Z_{d}}^{0}\right)^{2} \hat{Z}_{d}^{\mu} \hat{Z}_{d \mu}$,

where $\hat{B}_{\mu \nu}$ and $\hat{Z}_{d \mu \nu}$ are the field strengths of $\mathrm{U}(1)_{Y}$ in the $\mathrm{SM}$ and $U(1)_{D}$ in the dark sector, respectively, with a small mixing term parametrized by $\sin \varepsilon$, and $m_{Z_{d}}$ is the mass of dark gauge boson. Here, we assume that the hidden sector gauge symmetry is spontaneously broken by additional Higgs so that the mass of hidden gauge boson $Z_{d}$ is generated. The fermion dark matter $\chi$ has gauge interaction with hidden gauge boson with gauge coupling $g_{d}$ as 

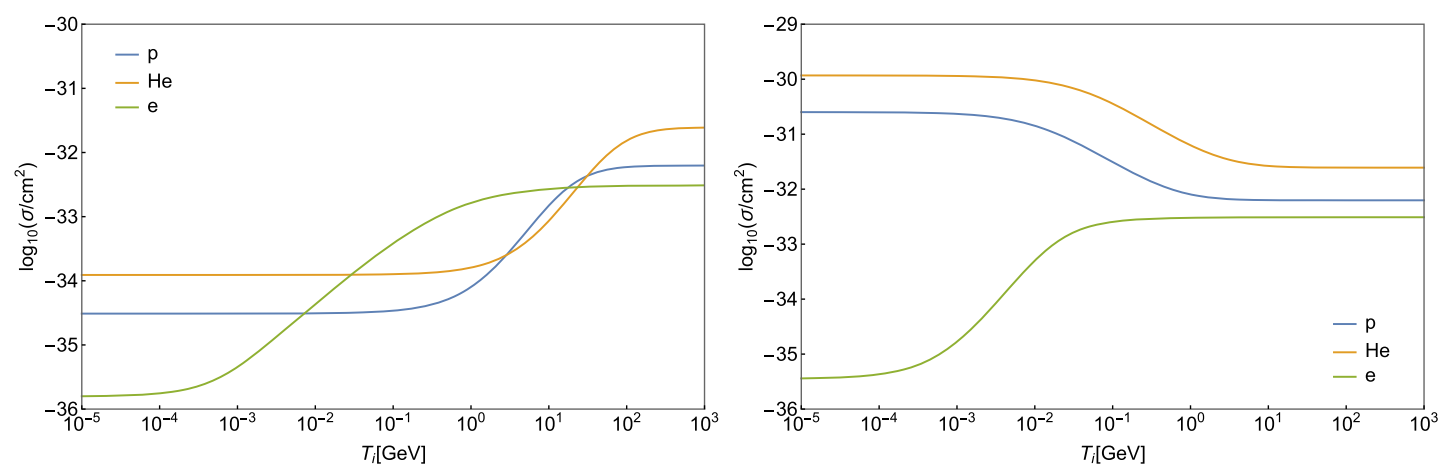

FIG. 1. The scattering cross section of DM and CRs in the DM rest frame with the kinetic energy $T_{i}$ of CR with DM mass $m_{\chi}=10^{-3} \mathrm{GeV}$ (left) and $0.1 \mathrm{GeV}$ (right). Here, we used $m_{Z d}=0.03 \mathrm{GeV}, \alpha_{d}=1$, and $\sin ^{2} \varepsilon=10^{-7}$.

$$
\mathcal{L}_{\mathrm{int}}=g_{d} \hat{Z}_{d \mu} \bar{\chi} \gamma^{\mu} \chi
$$

Below the electroweak symmetry breaking, the mass eigenstates (without hat) are related to the bare gauge fields (with hat) as

$$
\begin{aligned}
\hat{A} & =A_{\mathrm{SM}}-c_{W} t_{\varepsilon} s_{X} Z_{\mathrm{SM}}+c_{W} t_{\varepsilon} c_{X} Z_{d}, \\
\hat{Z} & =\left(c_{X}+s_{W} t_{\varepsilon} s_{X}\right) Z_{\mathrm{SM}}+\left(s_{X}-s_{W} t_{\varepsilon} c_{X}\right) Z_{d}, \\
\hat{Z}_{d} & =-\frac{s_{X}}{c_{\varepsilon}} Z_{\mathrm{SM}}+\frac{c_{X}}{c_{\varepsilon}} Z_{d},
\end{aligned}
$$

with the mixing angle $\theta_{X}$ given by

$$
\tan 2 \theta_{X}=\frac{2\left(m_{Z}^{0}\right)^{2} s_{W} t_{\varepsilon}}{\left(m_{Z}^{0}\right)^{2}\left(1-s_{W}^{2} t_{\varepsilon}^{2}\right)-\left(m_{Z_{d}}^{0}\right)^{2} / c_{\varepsilon}^{2}} .
$$

Here, $m_{Z}^{0}$ is the mass of $Z$ boson in the SM, and we use the abbreviations defined by $s_{W}=\sin \theta_{W}, c_{W}=\cos \theta_{W}$ with the Weinberg mixing angle $\theta_{W}$, and $t_{\varepsilon}=\tan \varepsilon, c_{\varepsilon}=\cos \varepsilon$, $s_{\varepsilon}=\sin \varepsilon$, and similarly for $c_{X}=\cos \theta_{X}$, and $s_{X}=\sin \theta_{X}$.

In the SM, the gauge interaction for a fermion $\psi$ with $\mathrm{SU}(2)$ charge $T_{3}$ and electromagnetic charge $Q$ is given by

$$
\mathcal{L}_{\mathrm{SM}, \mathrm{int}}=\bar{\psi} \gamma^{\mu} \psi\left\{e Q \hat{A}_{\mu}+\frac{e}{s_{W} c_{W}}\left(T_{3}-Q s_{W}^{2}\right) \hat{Z}_{\mu}\right\},
$$

where $\psi=\nu_{L}, e_{L}, e_{R}$, etc., and $e=|e|$. In the Appendix, we show the corresponding interaction Lagrangian between the DM and proton, neutron, electron, and neutrino, from which the elastic scattering cross sections are calculated.

For the scattering with the nucleus, the cross section at finite momentum transfer is corrected with a form factor as given by

$$
\sigma_{\chi N}\left(s, q^{2}\right)=\sigma_{\chi N}(s) \times F^{2}\left(q^{2}\right),
$$

where $q^{2}=2 m_{N} T_{N}$, with the mass of the target $m_{N}$ and recoil kinetic energy $T_{N}$. Here, we use the Helm form factor [30] with

$$
F\left(q^{2}\right)=3 \frac{j_{1}\left(q r_{n}\right)}{q r_{n}} e^{-q^{2} s^{2} / 2},
$$

where $j_{1}$ is the spherical Bessel function, $s=1 \mathrm{fm}$ is the nuclear skin thickness, and $r_{n}=\left(c^{2}+\frac{7}{3} \pi^{2} a^{2}-5 s^{2}\right)^{1 / 2}$ parametrizes the nuclear radius, with $c=1.23 A^{1 / 3}-$ $0.6 \mathrm{fm}$ and $a=0.52 \mathrm{fm}$, and $A$ is the mass number of the nucleus.

In Fig. 1, we show the total scattering cross sections in terms of the initial kinetic energy of CRs of the proton (blue), He (red), and electron (green), in the rest frame of DM with mass $m_{\chi}=10^{-3} \mathrm{GeV}$ (left) and $0.1 \mathrm{GeV}$ (right). Here, we used the parameters $m_{Z_{d}}=0.03 \mathrm{GeV}$, $\alpha_{d} \equiv g_{d}^{2} /(4 \pi)=1$, and $\sin ^{2} \varepsilon=10^{-7}$. We can see that the dependence of the cross section on $T_{i}$ varies for different mass parameters. When $m_{\chi}<m_{Z_{d}}$ (left), the cross section is enhanced at high $T_{i}$; however, when $m_{\chi}>m_{Z_{d}}$ (right), the cross section is suppressed at large $T_{i}$ due to the relations between the momentum transfer and the masses of the relevant particles.

\section{BOOSTED DARK MATTER FROM SCATTERINGS WITH COSMIC RAYS}

\section{A. Boosted DM}

The DMs in the galactic halo are scattered by the cosmic rays. In the initial rest frame of DM, the recoiled kinetic energy of DM after scattering $T_{\chi}$ can be written as

$$
\begin{aligned}
T_{\chi} & =T_{\chi}^{\max } \frac{1-\cos \theta}{2}, \\
T_{\chi}^{\max } & =\frac{T_{i}^{2}+2 m_{i} T_{i}}{T_{i}+\left(m_{\chi}+m_{i}\right)^{2} /\left(2 m_{\chi}\right)},
\end{aligned}
$$

where $m_{\chi}$ and $m_{i}$ are the mass of DM and the colliding CR particle, respectively, and $\theta$ is the scattering angle in the center-of-mass frame between the DM and CR particle. Here, $T_{\chi}^{\max }$ is the maximum kinetic energy that the DM can have after scattering. The mometum transfer in the 


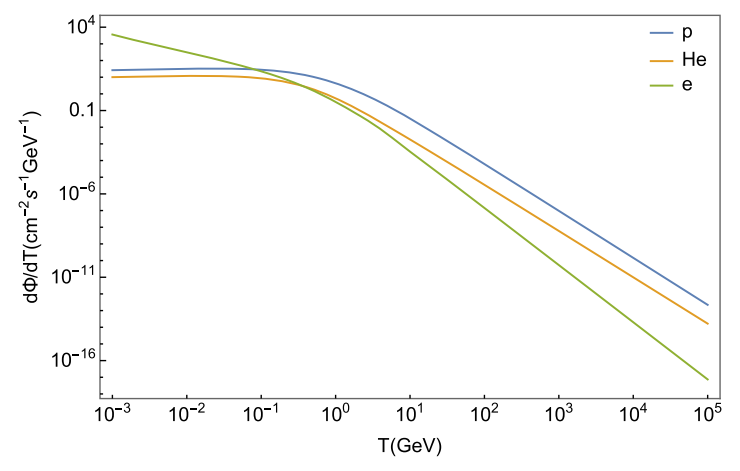

FIG. 2. Differential flux in terms of kinetic energy of the CR proton, helium, and electron [31].

collision can be written as $Q^{2}=2 m_{\chi} T_{\chi}$. In another way, the minimum kinetic energy of the cosmic particles to make DM with $T_{\chi}$ is given by

$T_{i}^{\min }=\left(\frac{T_{\chi}}{2}-m_{i}\right)\left(1 \pm \sqrt{1+\frac{2 T_{\chi}}{m_{\chi}} \frac{\left(m_{i}+m_{\chi}\right)^{2}}{\left(2 m_{i}-T_{\chi}\right)^{2}}}\right)$,

where + for $T_{\chi}>2 m_{i}$, and - for $T_{\chi}<2 m_{i}$. When DM collides with the nuclei in the rest frame, $i$ and $\chi$ are interchanged in the above equations.

To find the flux of BDM, we follow the method in Ref. [7]. The differential flux of BDM with the kinetic energy $T_{\chi}$ is obtained by integrating the flux of DM after scattering with an initial kinetic energy of cosmic particle $T_{i}$ as

$$
\begin{aligned}
\frac{d \Phi_{\chi}}{d T_{\chi}} & =\sum_{i=p, \mathrm{He}, e} \int_{T_{i}^{\min }}^{\infty} \mathrm{d} T_{i} \frac{d \Phi_{\chi}}{d T_{\chi} d T_{i}}, \\
& =\frac{\rho_{\chi}^{\text {local }}}{m_{\chi}} D_{\text {eff }} \sum_{i=p, \mathrm{He}, e, \nu} \int_{T_{i}^{\min }}^{\infty} \mathrm{d} T_{i} \frac{d \sigma_{\chi i}\left(T_{i}\right)}{d T_{\chi}} \frac{d \Phi_{i}^{\mathrm{LIS}}}{d T_{i}},
\end{aligned}
$$

where $T_{i}^{\text {min }}$ is the minimum energy of cosmic rays to give DM kinetic energy $T_{\chi}$ after collision. Here, we summed over the contributions from each $\mathrm{CR}$ of the proton, helium, and electron. In the second line, the scattering cross section between the DM and $\mathrm{CR} \sigma_{\chi i}$ is a function of $T_{i}$. For the flux of cosmic particles, we use the interstellar spectrum of the high energy cosmic particles observed by Voyger 1 [31]. In Fig. 2, we show the flux of CRs we used and assume that the CR flux is uniform in the DM halo.

In the second line, the effective distance $D_{\text {eff }}$ is defined as

$$
D_{\text {eff }}=\left(\rho_{\chi}^{\text {local }}\right)^{-1} \int \frac{d \Omega}{4 \pi} \int d \ell \rho_{\chi}
$$

where we used $\rho_{\chi}^{\text {local }}=0.3 \mathrm{GeV} / \mathrm{cm}^{3}$. In this paper, as a representative value, we use the effective distance $D_{\text {eff }}=1 \mathrm{kpc}$.

In Fig. 3, we show the flux of the BDM generated from scatterings with the proton (blue), He (orange), electron (green), and the total (black) for reference values of $m_{\chi}=0.1 \mathrm{GeV}, m_{Z_{d}}=30 \mathrm{MeV}, \alpha_{d}=1$, and $\sin ^{2} \varepsilon=10^{-7}$. For heavier DM with $m_{\chi}=0.1 \mathrm{GeV}$ (right), the proton and helium dominate; however, for the light DM with $m_{\chi}=1 \mathrm{MeV}$ (left), the electron scattering is comparable to those from the proton and helium. This can be easily understood from Fig. 1. When the mass of DM is lowered, the number of DM increases, and the cross section to nuclei is, however, decreased at $T_{i} \sim \mathrm{GeV}$, and they more or less compensate. However, for electron CR, the cross section is almost the same, and thus, the BDM flux increases for lighter DM. As can be seen from the Fig. 3 (left), with $m_{\chi}=10^{-3} \mathrm{GeV}$, the contribution of the CR proton and helium is dominant at $T_{\chi} \lesssim 0.1 \mathrm{GeV}$, while the electron contribution is larger at $T_{\chi} \gtrsim 0.1 \mathrm{GeV}$.

\section{B. Attenuation}

When the DMs come through the Earth's crust, they can interact with the medium and lose energy. This attenuation of kinetic energy could make DMs undetectable because the DMs cannot reach the detector, or the kinetic energy of

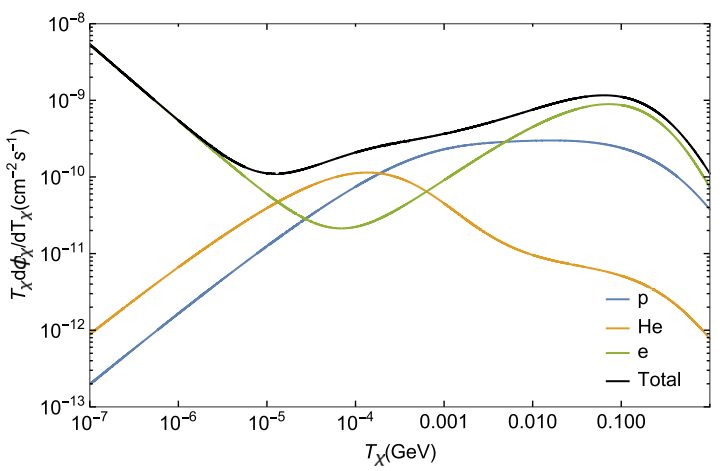

FIG. 3. Flux of BDM around Earth generated from scatterings with the proton (blue), He (orange), electron (green), and the total (black). Here, we used $m_{\chi}=10^{-3} \mathrm{GeV}$ (left), and $0.1 \mathrm{GeV}$ (right), with $m_{Z_{d}}=0.03 \mathrm{GeV}, \alpha_{d}=1$, and $\sin ^{2} \varepsilon=10^{-7}$. 


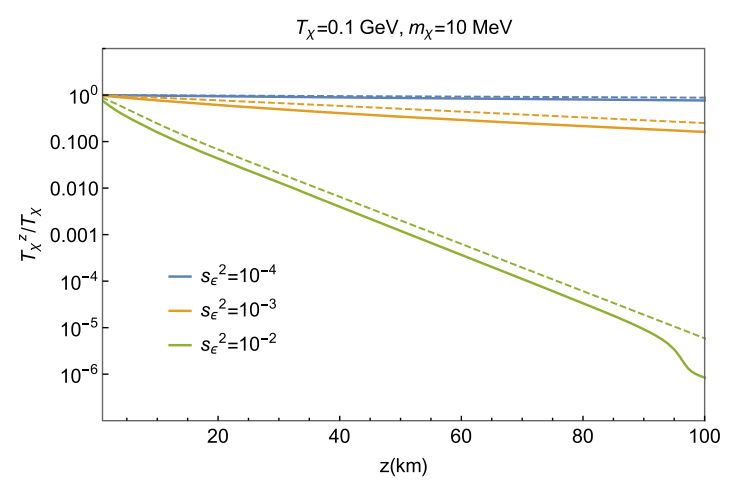

FIG. 4. The kinetic energy of BDM at depth $z$ below the Earth's surface due to the attenuation normalized by the initial kinetic energy. We show them for three cases of the mixing angles $\sin ^{2} \epsilon=10^{-2}, 10^{-3}, 10^{-4}$, with the green, red, and blue lines, respectively. The dashed lines show the attenuation with only nuclei ignoring electrons.

DM becomes too small for the threshold in the direct detection. The energy loss of DM particles per depth that pass through the medium is [7]

$$
\frac{d T_{\chi}}{d z}=-\sum_{N} n_{N} \int_{0}^{T_{r}^{\max }} \frac{d \sigma_{\chi N}\left(T_{r}\right)}{d T_{r}} T_{r} d T_{r},
$$

where $T_{r}$ is the energy lost by BDM in a collision with nucleus $N$. In a realistic model, the energy dependence of the cross section must be considered. By solving this differential equation, we can find the relation between the DM kinetic energies $T_{\chi}$ above the Earth and $T_{\chi}^{z}$ at depth $z$ below the Earth's surface. In Fig. 4, we show the change of the kinetic energy of BDM at depth $z$ in the Earth due to the attenuation with the nuclei and electron (solid) and nuclei alone without electron (dashed) for three cases of mixing with $\sin ^{2} \epsilon=10^{-2}, 10^{-3}, 10^{-4}$, with green, red, and blue lines, respectively. For low DM mass, the attenuation due to electron is also comparable to that from nuclei; however, for heavy DM, the attenuation is dominated by the collision with nuclei.

In a recent paper [32], they studied new diurnal effects from the boosted dark matter due to the anisotropic boosted DM flux and the Earth attenuation. However, in our paper, the effect is averaged out since we add up the events for a long period to compare with the experiments.

\section{DIRECT DETECTION OF BOOSTED DM}

The DMs that survived the attenuation of the Earth's crust reach the underground detector and can scatter the nuclei or the electrons.

\section{A. DM-nucleus interaction}

The BDMs that reach down to the Earth could collide with target nucleus inside in the detector [7]. This time, the nucleus is at rest, and the DM is moving, which is the opposite situation for upscattering DM by cosmic rays. The differential rate per target nucleus is obtained similarly to that of Eq. (10) as

$$
\frac{d \Gamma_{N}}{d T_{N}}=\int_{T_{\chi}\left(T_{\chi}^{\min , z}\right)}^{\infty} d T_{\chi} \frac{d \sigma_{\chi N}\left(T_{\chi}^{z}\right)}{d T_{N}} \frac{d \Phi_{\chi}}{d T_{\chi}},
$$

where the $T_{\chi}\left(T_{\chi}^{\mathrm{min}, \mathrm{z}}\right)$ is kinetic energy of boosted DM particle outside Earth, which gives the minimum kinetic energy to make kinetic energy of target nucleus $T_{N}$ at the depth $z$ inside the Earth. The scattering cross section is a function of the DM kinetic energy at the location of the detector $\sigma_{\chi N}\left(T_{\chi}^{z}\right)$, and here, $T_{\chi}^{z}$ is a function of $T_{\chi}$ after the attenuation in the Earth, which is evaluated from Eq. (12).

Then, we can calculate the number of the events $N_{\text {sig }}$ by integrating between the experimentally accessible recoil energies $T_{N} \in\left\{T_{1}, T_{2}\right\}$, for the corresponding observational time $\Delta t$ and target number $N_{T}$,

$$
N_{\text {sig }}=N_{T} \times \Delta t \times \int_{T_{1}}^{T_{2}} \frac{d \Gamma_{N}}{d T_{N}},
$$

and compare it with the observational constraint.

For the present bound, we use the DM search results from a 1 ton-year exposure of Xenon-1T [2], where 1.3 ton of xenon was exposed for 278.8 days with nuclear recoil energy region between $T_{1}=4.9 \mathrm{keV}$ and $T_{2}=40.9 \mathrm{keV}$, and there was no excess found over the background, and thus, we require that $N_{\text {sig }}<754$. For future prospects, we use factor of 10 for higher sensitivity with Xenon-nT [33] and 500 to get to the neutrino floor.

\section{B. DM-electron interaction}

The BDM scatterings with electron can be probed if the recoil energy of the electron $T_{e}$ is large enough [8]. Using the results of Super-Kamiokande (Super- $K$ ) with 161.9 kton $\mathrm{yr}$ [34] that is searching signals in the range $0.1 \mathrm{GeV}<T_{e}<1.33 \mathrm{GeV}$, we apply that the number of the events is smaller than 4042 for 2628.1 days of Super- $K$ to put the constraint.

\section{Results}

In Fig. 5, we show the constraints on the parameters of $\left(m_{\chi}, \sin ^{2} \varepsilon\right)$ from BDM for the fixed values of $\alpha_{d}=1$ and $m_{Z_{d}}=30 \mathrm{MeV}$. The red (blue) shaded region in the left top is disallowed from the direct detection of the BDM with nuclei (electrons) in the detector. The future prospects are also shown with dashed (10 times) dotted lines (500 times). The constraints from other experiments are shown with thin colors: direct detection with nuclei (orange) [35], direct detection with electrons (green) [21], and astrophysics and cosmology (gray). 


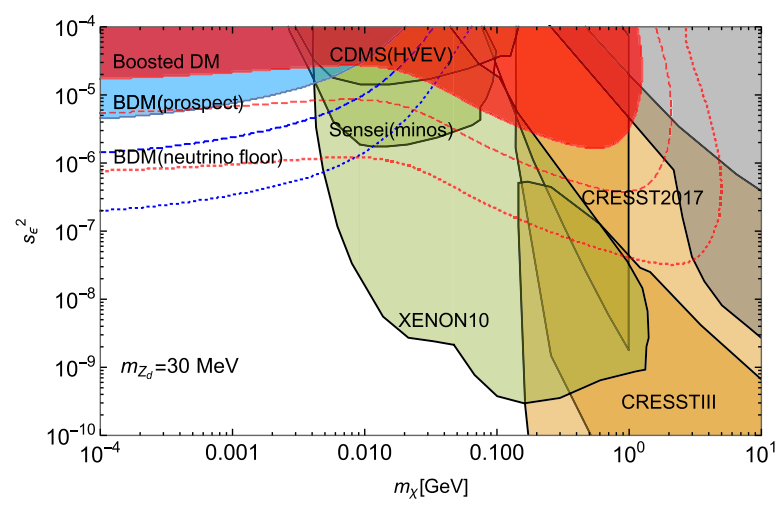

FIG. 5. Constraints on the DM mass and kinetic mixing from BDM through the scatterings with nuclei (red) and electrons (blue). Here, we used $\alpha_{d}=1$ and $m_{Z_{d}}=30 \mathrm{MeV}$. The future prospects are shown with dashed and dotted lines, The constraints from other direct detection experiments and astrophysical observations are also shown with thin colors.

The BDM constraints complements the other bounds of the direct detection with the nonrelativistic DM. This new bound closes a small open spot at around $m_{\chi}=0.1 \mathrm{GeV}$ and $\sin ^{2} \varepsilon=3 \times 10^{-5}$ and excludes the region of $m_{\chi}<$ $4 \mathrm{MeV}$ and $\sin ^{2} \varepsilon \gtrsim 10^{-5}$, which is not probed by nonrelativistic DM direct detection. However, the white region of the left bottom is also constrained when we include the bound from the beam-dump experiments.

In this realistic model of DM, the shape of the constraint is different from those where constant cross section was assumed [7-9] or that where a simple vector mediation model to the nucleon was used [10]. The $m_{\chi}$ dependence of the BDM constraint can be understood as follows.

First, the number of DM in the halo is inversely proportional to $m_{\chi}$. For the DM-nucleus direct detection, we need to have recoil energy of nucleus larger than $\mathrm{keV}$. For $m_{\chi} \lesssim 10 \mathrm{MeV}$, this is satisfied for the DM kinetic energy larger than around $10 \mathrm{MeV}$, at which the BDM flux is mainly from $\mathrm{CR}$ of the proton and helium as well as comparable contribution from the electron. The energy transferred from CR proton to DM scales as $T_{\chi} \simeq 2 m_{\chi} T_{i}^{2} / m_{p}^{2}$, and the integral of the CR flux, which scales $\sim T_{i}^{-2.7}$, is proportional to $\left(T_{i}^{\min }\right)^{-1.7} \propto m_{\chi}{ }^{0.85}$. Therefore, the event rate is proportional to $\Gamma \propto m_{\chi}{ }^{-1} \varepsilon^{4} m_{\chi}{ }^{0.85}=\varepsilon^{4} m_{\chi}{ }^{-0.15}$, which gives $\varepsilon^{2} \propto m_{\chi}{ }^{0.075}$. For $m_{\chi} \gtrsim 10 \mathrm{MeV}$, the $\mathrm{CR}$ proton to $\mathrm{DM}$ scattering cross section becomes dependent on $\varepsilon^{2} m_{\chi}{ }^{2}$, and also the recoil energy of the nucleus scales $T_{N} \simeq \frac{2 m_{\chi_{\chi}} T_{\chi}}{m_{N}}$, with $T \chi \simeq 2 m_{\chi} T_{i}^{2} / m_{p}^{2}$. Therefore, $\Gamma \propto \varepsilon^{4} m_{\chi}^{2.7}$, which gives $\varepsilon^{2} \propto m_{\chi}{ }^{-1.35}$. That explains the ups and downs of the BDM constraint (red) in Fig. 5.

For DM-electron direct detection in Super- $K$, it is necessary that the recoil energy of electron be larger than $100 \mathrm{MeV}$. For $m_{\chi} \lesssim 10 \mathrm{MeV}$, the dominant contribution to BDM comes from CR electron, and for $m_{\chi} \gtrsim 10 \mathrm{MeV}$, it comes from the CR proton or helium. For the low $m_{\chi}$

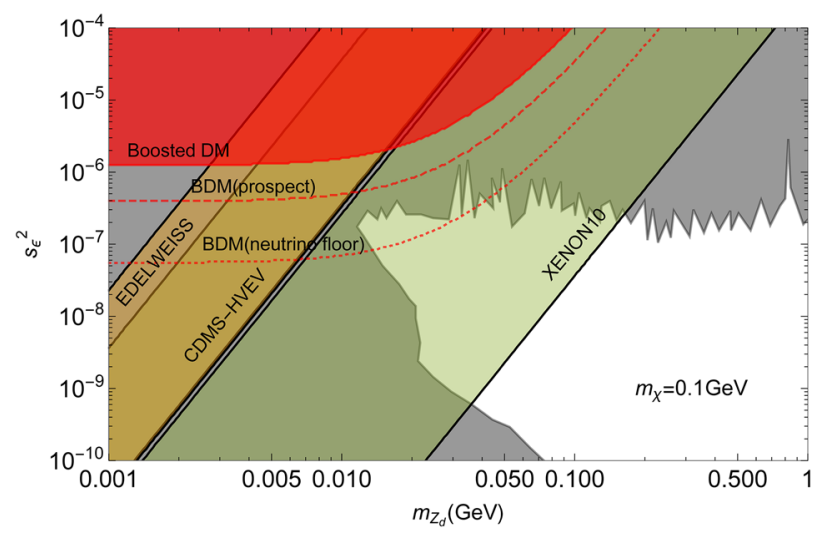

FIG. 6. Constraints from BDM on the parameter region $\left(m_{Z_{d}}, \sin ^{2} \varepsilon^{2}\right)$ with DM mass $m_{\chi}=100 \mathrm{MeV}$ for $\alpha_{d}=1$. Here, we used the constraint from Xenon-1T (solid), future prospect (dashed), and neutrino floor (dotted) [33]. The other constraints shown with thin colors include those from the collider and beam dump. The constraints from direct detection are also shown with orange and green colors.

region, the event rate scales as $\Gamma \propto m_{\chi}{ }^{-1} \varepsilon^{4}$, resulting in $\varepsilon^{2} \propto \sqrt{m_{\chi}}$. For the large $m_{\chi}$ region, $T_{e} \simeq 2 m_{e} T_{\chi}^{2} / m_{\chi}^{2}$, and $\Gamma \propto \varepsilon^{4} m_{\chi}{ }^{-2.7}$. This gives $\varepsilon^{2} \propto m_{\chi}{ }^{1.35}$ [8].

In Fig. 6, we show the constraints on the plane of $\left(m_{Z_{d}}, \sin ^{2} \varepsilon\right)$ for $m_{\chi}=100 \mathrm{MeV}$ and $\alpha_{d}=1$, with other direct detection bound (orange and green) as well as the constraints from collider [36] and beam-dump experiments [37] (gray). The present BDM constraint is already within the bounds of the collider, and, in the future, BDM may touch the unbounded region by them, though it is already ruled out by the Xenon-10 experiment.

\section{ASTROPHYSICAL CONSTRAINTS}

The large kinetic mixing of the hidden gauge boson with the SM may change the effective number of neutrinos, which represents the degrees of freedom of relativistic decoupled species. The current Planck observation gives a lower bound on the allowed mass of hidden gauge boson around $8.5 \mathrm{MeV}$ for the mixing parameter $\sin \varepsilon \gtrsim 10^{-9}$ [22].

The large annihilation of DMs in the early Universe also can affect the Big Bang Nucleosynthesis and Cosmic Microwave Background [26,38]. However, this may be avoided for a specific models of dark matter, such as asymmetric dark matter. This requires nonthermal production of dark matter, which is beyond of our simple model of kinetic mixing [39].

\section{CONCLUSION}

We studied the impact of the boosted dark matter generated by scatterings of the high energy cosmic rays mediated by the U(1) gauge kinetic mixing. The nonobservation in the underground direct detection combined with the BDM constrains the light dark matter region, 
independently of the previous bounds of the direct detection, as well as the collider and beam-dump experiments.

\section{ACKNOWLEDGMENTS}

The authors were supported by the National Research Foundation of Korea (NRF) Grant funded by the Korea government (MEST) (NRF-2019R1A2B5B01070181).

\section{APPENDIX: KINEMATICS AND THE CROSS SECTION}

\section{Kinematics}

The differential cross section for elastic scattering of particle 1 and 2 is given by

$$
\frac{d \sigma}{d t}=\frac{|\overline{\mathcal{M}}|^{2}}{16 \pi \lambda\left(s, m_{1}^{2}, m_{2}^{2}\right)},
$$

where $\lambda(a, b, c)=a^{2}+b^{2}+c^{2}-2 a b-2 b c-2 c a=$ $\left[a-(\sqrt{b}+\sqrt{c})^{2}\right]\left[a-(\sqrt{b}-\sqrt{c})^{2}\right]$. If particle 2 is at rest initially, the Mandelstam variables are given by

$$
\begin{aligned}
s & =m_{1}^{2}+m_{2}^{2}+2 E_{1} m_{2}, \\
& =\left(m_{1}+m_{2}\right)^{2}+2 T_{1} m_{2}=M^{2}+2 m_{1} m_{2}+2 T_{1} m_{2}, \\
t & =2 m_{2}^{2}-2 m_{2} E_{2}=-2 m_{2} T_{2}, \\
u & =2\left(m_{1}^{2}+m_{2}^{2}\right)-s-t=M^{2}-2 m_{1} m_{2}-2 m_{2}\left(T_{1}-T_{2}\right),
\end{aligned}
$$

where $M^{2}=m_{1}^{2}+m_{2}^{2}$, and

$$
\begin{aligned}
\lambda\left(s, m_{1}^{2}, m_{2}^{2}\right) & =\left(s-\left(m_{1}+m_{2}\right)^{2}\right)\left(s-\left(m_{1}-m_{2}\right)^{2}\right) \\
& =\left(2 E_{1} m_{2}-2 m_{1} m_{2}\right)\left(2 E_{1} m_{2}+2 m_{1} m_{2}\right) \\
& =4 m_{2}^{2}\left(T_{1}^{2}+2 m_{1} T_{1}\right) \\
& =2 s \cdot m_{2} \cdot T_{2}^{\max } .
\end{aligned}
$$

Here, $T_{1}$ is the kinetic energy of a particle 1 before collision, and $T_{2}$ is the kinetic energy of a particle 2 after collision, with the maximum of $T_{2}$ given by

$$
T_{2}^{\max }=\frac{T_{1}^{2}+2 m_{1} T_{1}}{T_{1}+\left(m_{1}+m_{2}\right)^{2} /\left(2 m_{2}\right)} .
$$

Therefore, we can write Eq. (A1) into

$$
\frac{d \sigma}{d T_{2}}=-2 m_{2} \frac{d \sigma}{d t}=-\frac{|\overline{\mathcal{M}}|^{2}}{16 \pi s T_{2}^{\max }} .
$$

If $|\overline{\mathcal{M}}|^{2}$ is constant, the total cross section becomes

$$
\sigma_{\mathrm{tot}}=\int_{-2 m_{2} T_{2}^{\max }}^{0}\left(\frac{d \sigma}{d t}\right) \mathrm{d} t=\frac{|\overline{\mathcal{M}}|^{2}}{16 \pi s}
$$

\section{Scattering cross section of DM in the model of dark gauge boson}

The Lagrangian we are using is written by

$\mathcal{L}=\mathcal{L}_{\mathrm{SM}}-\frac{1}{4} \hat{Z}_{d \mu \nu} \hat{Z}_{d}^{\mu \nu}+\frac{\sin \varepsilon}{2} \hat{B}_{\mu \nu} \hat{Z}_{d}^{\mu \nu}+\frac{1}{2}\left(m_{Z_{d}}^{0}\right)^{2} \hat{Z}_{d}^{\mu} \hat{Z}_{d \mu}+\mathcal{L}_{\text {int }}$,

where $\hat{B}_{\mu \nu}$ and $\hat{Z}_{d \mu \nu}$ are the field strengths of $\mathrm{U}(1)_{Y}$ in the $\mathrm{SM}$ and $\mathrm{U}(1)_{D}$ in the dark sector, respectively, with a small mixing term parametrized by $\sin \varepsilon$, and $m_{Z_{d}}$ is the mass of dark photon. The fermion dark matter $\chi$ has a gauge interaction with the gauge boson with gauge coupling $g_{d}$ as

$$
\mathcal{L}_{\text {int }}=g_{d} \bar{\chi} \gamma^{\mu} \chi \hat{Z}_{d \mu}
$$

The mixing term between $\hat{B}$ and $\hat{Z}_{d}$ can be removed by the field redefinition,

$$
\left[\begin{array}{c}
B_{\mu}^{0} \\
Z_{d \mu}^{0}
\end{array}\right]=\left[\begin{array}{cc}
1 & -\sin \varepsilon \\
0 & \cos \varepsilon
\end{array}\right]\left[\begin{array}{c}
\hat{B}_{\mu} \\
\hat{Z}_{d \mu}
\end{array}\right]
$$

The electroweak symmetry breaking generates mass to $\hat{Z}$ boson with massless $\hat{A}$, which are defined by

$\hat{A}_{\mu}=c_{W} \hat{B}_{\mu}+s_{W} \hat{W}_{\mu}^{3}, \quad \hat{Z}_{\mu}=-s_{W} \hat{B}_{\mu}+c_{W} \hat{W}_{\mu}^{3}$,

in terms of the Weinberg mixing angle $\theta_{W}$, with $c_{W} \equiv$ $\cos \theta_{W}$ and $s_{W}=\sin \theta_{W}$. The mass term can be written in terms of $Z^{0}$ and $Z_{d}^{0}$ by

$$
\begin{aligned}
\frac{1}{2}\left(m_{Z}^{0}\right)^{2} \hat{Z}_{\mu} \hat{Z}^{\mu}= & \frac{1}{2}\left(m_{Z}^{0}\right)^{2}\left(-s_{W} \hat{B}_{\mu}+c_{W} \hat{W}_{\mu}^{3}\right)\left(-s_{W} \hat{B}^{\mu}+c_{W} \hat{W}^{3, \mu}\right), \\
= & \frac{1}{2}\left(m_{Z}^{0}\right)^{2} Z_{\mu}^{0} Z^{0, \mu}-\left(m_{Z}^{0}\right)^{2} s_{W} t_{\varepsilon} Z_{\mu}^{0} Z_{d}^{0, \mu} \\
& +\frac{1}{2}\left(m_{Z}^{0}\right)^{2} s_{W}^{2} t_{\varepsilon}^{2} Z_{d \mu}^{0} Z_{d}^{0, \mu}
\end{aligned}
$$

where

$$
Z_{\mu}^{0}=-s_{W} B_{\mu}^{0}+c_{W} \hat{W}_{\mu}^{3}, \quad A_{\mu}^{0}=\hat{A}_{\mu} .
$$

Then, the mass matrix in the basis of $\left(A^{0}, Z^{0}, Z_{d}^{0}\right)$ is written as

$M^{2}=\left[\begin{array}{ccc}1 & 0 & 0 \\ 0 & \left(m_{Z}^{0}\right)^{2} & -\left(m_{Z}^{0}\right)^{2} s_{W} t_{\varepsilon} \\ 0 & -\left(m_{Z}^{0}\right)^{2} s_{W} t_{\varepsilon} & \frac{\left(m_{Z_{d}}^{0}\right)^{2}}{\cos ^{2} \varepsilon}+\left(m_{Z}^{0}\right)^{2} s_{W}^{2} t_{\varepsilon}^{2}\end{array}\right]$,

which can be diagonalized to find the mass eigenstates $\left(A_{\mathrm{SM}}, Z_{\mathrm{SM}}, Z_{d}\right)$ 


$$
\left[\begin{array}{c}
A_{\mathrm{SM} \mu} \\
Z_{\mathrm{SM} \mu} \\
Z_{d \mu}
\end{array}\right]=\left[\begin{array}{ccc}
1 & 0 & 0 \\
0 & \cos \theta_{X} & -\sin \theta_{X} \\
0 & \sin \theta_{X} & \cos \theta_{X}
\end{array}\right]\left[\begin{array}{c}
A_{\mu}^{0} \\
Z_{\mu}^{0} \\
Z_{d \mu}^{0}
\end{array}\right],
$$

with the mixing angle $\theta_{X}$ given by

$$
\tan 2 \theta_{X}=\frac{2\left(m_{Z}^{0}\right)^{2} s_{W} t_{\varepsilon}}{\left(m_{Z}^{0}\right)^{2}\left(1-s_{W}^{2} t_{\varepsilon}^{2}\right)-\left(m_{Z_{d}}^{0}\right)^{2} / c_{\varepsilon}^{2}}
$$

The mass eigenvalues for $Z_{\mathrm{SM}}$ and $Z_{d}$ are [24]

$$
\begin{aligned}
m_{Z_{\mathrm{SM}}}^{2} & =\left(m_{Z}^{0}\right)^{2}\left(1+s_{W} t_{\varepsilon} t_{X}\right), \\
m_{Z_{d}}^{2} & =\frac{\left(m_{Z_{d}}^{0}\right)^{2}}{c_{\varepsilon}^{2}}\left(1+s_{W} t_{\varepsilon} t_{X}\right)^{-1} .
\end{aligned}
$$

In this paper, with small $\varepsilon$, we can approximate $m_{Z_{\mathrm{SM}}} \simeq m_{Z}^{0}$ and $m_{Z_{d}} \simeq m_{Z_{d}}^{0}$. By rearranging the above terms, we can find the relations between the mass eigenstates of the gauge bosons $\left(A_{\mathrm{SM}}, Z_{\mathrm{SM}}, Z_{d}\right)$ and the interaction eigenstates $\left(\hat{A}, \hat{Z}, \hat{Z}_{d}\right)$ as

$$
\begin{aligned}
\hat{A} & =A_{\mathrm{SM}}-c_{W} t_{\varepsilon} s_{X} Z_{\mathrm{SM}}+c_{W} t_{\varepsilon} c_{X} Z_{d}, \\
\hat{Z} & =\left(c_{X}+s_{W} t_{\varepsilon} s_{X}\right) Z_{\mathrm{SM}}+\left(s_{X}-s_{W} t_{\varepsilon} c_{X}\right) Z_{d}, \\
\hat{Z}_{d} & =-\frac{s_{X}}{c_{\varepsilon}} Z_{\mathrm{SM}}+\frac{c_{X}}{c_{\varepsilon}} Z_{d} .
\end{aligned}
$$

For the Standard Model, the gauge interaction for a fermion $\psi$ with $\mathrm{SU}(2)$ charge $T_{3}$ and EM charge $Q$ is

$$
\mathcal{L}_{\text {int }}=\bar{\psi} \gamma^{\mu} \psi\left\{e Q \hat{A}_{\mu}+\frac{e}{s_{W} c_{W}}\left(T_{3}-Q s_{W}^{2}\right) \hat{Z}_{\mu}\right\}
$$

where $\psi=\nu_{L}, e_{L}, e_{R}$, etc. and $e=|e|$. By using Eq. (A17), we can find easily the interaction of SM particles to the mass eigenstates of the gauge bosons.

\section{DM-electron scattering}

The interaction Lagrangian of electron is given by

$$
\begin{aligned}
\mathcal{L}_{\text {int }}= & \overline{\mathbf{e}} \gamma^{\mu} \mathbf{e}\left[-e A_{\mathrm{SM} \mu}+g_{C} Z_{\mathrm{SM} \mu}+g_{C d} Z_{d \mu}\right] \\
& +\overline{\mathbf{e}} \gamma^{\mu} \gamma^{5} \mathbf{e}\left[g_{A} Z_{\mathrm{SM} \mu}+g_{A d} Z_{d \mu}\right],
\end{aligned}
$$

where

$$
\begin{aligned}
g_{C} & =\frac{e}{4}\left[c_{X}\left(3 \tan \theta_{W}-\cot \theta_{W}\right)+\frac{3 s_{X} t_{\varepsilon}}{c_{W}}\right], \\
g_{C d} & =\frac{e}{4}\left[s_{X}\left(3 \tan \theta_{W}-\cot \theta_{W}\right)-\frac{3 c_{X} t_{\varepsilon}}{c_{W}}\right], \\
g_{A} & =\frac{e}{4 c_{W}}\left[\frac{c_{X}}{s_{W}}+s_{X} t_{\varepsilon}\right], \\
g_{A d} & =\frac{e}{4 c_{W}}\left[\frac{s_{X}}{s_{W}}-c_{X} t_{\varepsilon}\right] .
\end{aligned}
$$

Note that $t_{X} \simeq s_{W} t_{\varepsilon}\left(1-m_{Z_{d}}{ }^{2} / m_{Z}^{2}\right)^{-1}$ for very small $\varepsilon$ and $\theta_{X}$, and thus $g_{C d}$ and $g_{A d}$ become

$$
\begin{aligned}
g_{C d} & \sim \frac{e}{4} \frac{m_{Z_{d}}^{2}}{m_{Z}^{2}-m_{Z_{d}}^{2}} \frac{c_{W}^{2}-3 s_{W}^{2}}{c_{W}} \varepsilon, \\
g_{A d} & \sim \frac{e}{4} \frac{m_{Z_{d}}^{2}}{m_{Z}^{2}-m_{Z_{d}}^{2}} \frac{1}{c_{W}} \varepsilon .
\end{aligned}
$$

The invariant matrix element $\mathcal{M}$ is

$$
\begin{aligned}
i \mathcal{M}= & \bar{u}_{p_{\chi}}^{s}\left(i g_{d} \frac{s_{X}}{\sqrt{1-\varepsilon^{2}}} \gamma^{\mu}\right) u_{k_{\chi}}^{s^{\prime}}\left[\frac{-i\left(\eta_{\mu \nu}-\frac{q_{\mu} q_{\nu}}{m_{Z}^{2}}\right)}{q^{2}-m_{Z}^{2}}\right] \\
& \times \bar{u}_{p_{e}}^{r}\left(i \gamma^{\nu}\left(g_{C}+g_{A} \gamma^{5}\right)\right) u_{k_{e}}^{r^{\prime}} \\
& +\bar{u}_{p_{\chi}}^{s}\left(-i g_{d} \frac{c_{X}}{\sqrt{1-\varepsilon^{2}}} \gamma^{\mu}\right) u_{k_{\chi}}^{s^{\prime}}\left[\frac{-i\left(\eta_{\mu \nu}-\frac{q_{\mu} q_{\nu}}{m_{Z_{d}}^{2}}\right)}{q^{2}-m_{Z_{d}}^{2}}\right] \\
& \times \bar{u}_{p_{e}}^{r}\left(i \gamma^{v}\left(g_{C d}+g_{A d} \gamma^{5}\right)\right) u_{k_{e}}^{r^{\prime}}
\end{aligned}
$$

and the spin-averaged amplitude squared is

$$
\begin{aligned}
\overline{|\mathcal{M}|^{2}}= & \frac{2 g_{d}^{2}}{1-\varepsilon^{2}}\left[\left(\frac{s_{X} g_{C}}{t-m_{Z}^{2}}-\frac{c_{X} g_{C d}}{t-m_{Z_{d}}^{2}}\right)^{2} A\left(m_{\chi}, m_{e}\right)\right. \\
& \left.+\left(\frac{s_{X} g_{A}}{t-m_{Z}^{2}}-\frac{c_{X} g_{A d}}{t-m_{Z_{d}}^{2}}\right)^{2} B\left(m_{\chi}, m_{e}\right)\right],
\end{aligned}
$$

where

$$
\begin{aligned}
A\left(m_{\chi}, m_{i}\right) & =2 t M^{2}+\left(s-M^{2}\right)^{2}+\left(u-M^{2}\right)^{2}, \\
B\left(m_{\chi}, m_{i}\right) & =\left(s-M^{2}\right)^{2}+\left(u-M^{2}\right)^{2}+2 t\left(m_{\chi}^{2}-m_{i}^{2}\right)-8 m_{\chi}^{2} m_{i}^{2}, \\
\text { with } M^{2} & =m_{\chi}^{2}+m_{i}^{2} .
\end{aligned}
$$

For the nonrelativistic limit, $s \rightarrow\left(m_{1}+m_{2}\right)^{2}, t \rightarrow 0$, and $u \rightarrow\left(m_{1}-m_{2}\right)^{2}$, then $A\left(m_{\chi}, m_{i}\right)=8 m_{\chi}^{2} m_{i}^{2}$, and $B\left(m_{\chi}, m_{i}\right)=$ 0 . In this limit, Eq. (A2) becomes

$$
\begin{aligned}
t & =-2 m_{2} T_{2}, \\
s-M^{2} & =2 m_{1} m_{2}+2 m_{2} T_{1}, \\
u-M^{2} & =-2 m_{1} m_{2}-2 m_{2}\left(T_{1}-T_{2}\right) .
\end{aligned}
$$


For the nonrelativistic limit, $\overline{|\mathcal{M}|^{2}}$ becomes

$$
\overline{|\mathcal{M}|^{2}}=\frac{16 g_{d}^{2} m_{\chi}^{2} m_{e}^{2}}{1-\varepsilon^{2}}\left(\frac{s_{X} g_{C}}{m_{Z}^{2}}-\frac{c_{X} g_{C d}}{m_{Z_{d}}^{2}}\right)^{2},
$$

and the scattering cross section is given by

$$
\sigma_{\chi e}^{\mathrm{NR}}=\frac{g_{d}^{2} \mu_{\chi e}^{2}}{\pi\left(1-\varepsilon^{2}\right)}\left(\frac{s_{X} g_{C}}{m_{Z}^{2}}-\frac{c_{X} g_{C d}}{m_{Z_{d}}^{2}}\right)^{2} .
$$

\section{DM-neutrino scattering}

The interaction Lagrangian of neutrino is given by

$$
\mathcal{L}_{\text {int }}=\bar{\nu}_{e} \gamma^{\mu}\left(1-\gamma^{5}\right)\left[g_{A} Z_{\mathrm{SM} \mu}+g_{A d} Z_{d \mu}\right] \nu_{\mathbf{e}}
$$

Within the invariant matrix element, $\mathcal{M}$ is given by

$$
\begin{aligned}
i \mathcal{M}= & \bar{\chi}\left(p^{\prime}\right)\left(i g_{d} \frac{s_{X}}{\sqrt{1-\varepsilon^{2}}} \gamma^{\mu}\right) \chi(p)\left[\frac{-i\left(\eta_{\mu \nu}-\frac{q_{\mu} q_{\nu}}{m_{Z}^{2}}\right)}{q^{2}-m_{Z}^{2}}\right] \bar{\nu}_{e}\left(k^{\prime}\right)\left(-i g_{A} \gamma^{\nu}\left(1-\gamma^{5}\right)\right) \nu_{e}(k) \\
& +\bar{\chi}\left(p^{\prime}\right)\left(-i g_{d} \frac{c_{X}}{\sqrt{1-\varepsilon^{2}}} \gamma^{\mu}\right) \chi(p)\left[\frac{-i\left(\eta_{\mu \nu}-\frac{q_{\mu} q_{\nu}}{m_{Z_{d}}^{2}}\right)}{q^{2}-m_{Z_{d}}^{2}}\right] \bar{\nu}_{e}\left(k^{\prime}\right)\left(-i g_{A d} \gamma^{v}\left(1-\gamma^{5}\right)\right) \nu_{e}(k),
\end{aligned}
$$

and the spin-averaged amplitude squared is obtained as

$$
\overline{|\mathcal{M}|^{2}}=\frac{4 g_{d}^{2} A\left(m_{\chi}, 0\right)}{\left(1-\varepsilon^{2}\right)}\left(\frac{s_{X} g_{A}}{\left(t-m_{Z}^{2}\right)}-\frac{c_{X} g_{A d}}{\left(t-m_{Z_{d}}^{2}\right)}\right)^{2} .
$$

\section{DM-nucleus scattering}

The interaction Lagrangian of the proton and neutron is given by

$$
\mathcal{L}_{\text {int }}=\overline{\mathbf{p}} \gamma^{\mu} \mathbf{p}\left(e A_{\mathrm{SM} \mu}-g_{C} Z_{\mathrm{SM} \mu}-g_{C d} Z_{d \mu}\right)+\overline{\mathbf{p}} \gamma^{\mu} \gamma^{5} \mathbf{p}\left(-g_{A} Z_{\mathrm{SM} \mu}-g_{A d} Z_{d \mu}\right)+\overline{\mathbf{n}} \gamma^{\mu}\left(1-\gamma^{5}\right) \mathbf{n}\left(-g_{A} Z_{\mathrm{SM} \mu}-g_{A d} Z_{d \mu}\right),
$$

and thus, the interaction of the nucleus with mass number $A$ and the number of proton $Z$ is

$$
\begin{aligned}
\mathcal{L}_{\text {int }}=\overline{\mathbf{N}} \gamma^{\mu} \mathbf{N}\left[Z e A_{\mathrm{SM} \mu}-g_{\mathrm{NC}} Z_{\mathrm{SM} \mu}-g_{\mathrm{NC} d} Z_{d \mu}\right]+\overline{\mathbf{N}} \gamma^{\mu} \gamma^{5} \mathbf{N}\left[-g_{\mathrm{NA}} Z_{\mathrm{SM} \mu}-g_{N A d} Z_{d \mu}\right] \\
g_{\mathrm{NC}}=Z g_{C}+(A-Z) g_{A}, \\
g_{\mathrm{NC} d}=Z g_{C d}+(A-Z) g_{A d}, \\
g_{\mathrm{NA}}=(2 Z-A) g_{A}, \\
g_{\mathrm{NAd}}=(2 Z-A) g_{A d} .
\end{aligned}
$$

The invariant matrix element $\mathcal{M}$ is

$$
\begin{aligned}
i \mathcal{M}= & \bar{u}_{p_{\chi}}^{s}\left(i g_{d} \frac{s_{X}}{\sqrt{1-\varepsilon^{2}}} \gamma^{\mu}\right) u_{k_{\chi}^{s^{\prime}}}\left[\frac{-i\left(\eta_{\mu \nu}-\frac{q_{\mu} q_{\nu}}{m_{Z}^{2}}\right)}{q^{2}-m_{Z}^{2}}\right] \bar{u}_{p_{N}}^{r}\left(-i \gamma^{\nu}\left(g_{\mathrm{NC}}+g_{\mathrm{NA}} \gamma^{5}\right)\right) u_{k_{N}}^{r^{\prime}} \\
& +\bar{u}_{p_{\chi}}^{s}\left(-i g_{d} \frac{c_{X}}{\sqrt{1-\varepsilon^{2}}} \gamma^{\mu}\right) u_{k_{\chi}}^{s^{\prime}}\left[\frac{-i\left(\eta_{\mu \nu}-\frac{q_{\mu} q_{\nu}}{m_{Z_{d}}^{2}}\right)}{q^{2}-m_{Z_{d}}^{2}}\right] \bar{u}_{p_{N}}^{r}\left(-i \gamma^{v}\left(g_{\mathrm{NC} d}+g_{\mathrm{NA} d} \gamma^{5}\right)\right) u_{k_{N}}^{r^{\prime}},
\end{aligned}
$$

and the spin-averaged amplitude squared is

$$
\overline{|\mathcal{M}|^{2}}=\frac{2 g_{d}^{2}}{1-\varepsilon^{2}}\left[\left(\frac{s_{X} g_{\mathrm{NC}}}{t-m_{Z}^{2}}-\frac{c_{X} g_{\mathrm{NC} d}}{t-m_{Z_{d}}^{2}}\right)^{2} A\left(m_{\chi}, m_{N}\right)+\left(\frac{s_{X} g_{\mathrm{NA}}}{t-m_{Z}^{2}}-\frac{c_{X} g_{\mathrm{NA} d}}{t-m_{Z_{d}}^{2}}\right)^{2} B\left(m_{\chi}, m_{N}\right)\right] \text {. }
$$


For the nonrelativistic limit, it becomes

$$
\begin{aligned}
\overline{|\mathcal{M}|^{2}} & =\frac{16 g_{d}^{2} m_{\chi}^{2} m_{N}^{2}}{1-\varepsilon^{2}}\left(\frac{s_{X} g_{\mathrm{NC}}}{m_{Z}^{2}}-\frac{c_{X} g_{\mathrm{NC} d}}{m_{Z_{d}}^{2}}\right)^{2} \\
& =\frac{16 g_{d}^{2} m_{\chi}^{2} m_{N}^{2}}{1-\varepsilon^{2}}\left(Z\left(\frac{s_{X} g_{C}}{m_{Z}^{2}}-\frac{c_{X} g_{C d}}{m_{Z_{d}}^{2}}\right)+(A-Z)\left(\frac{s_{X} g_{A}}{m_{Z}^{2}}-\frac{c_{X} g_{A d}}{m_{Z_{d}}^{2}}\right)\right)^{2}
\end{aligned}
$$

and the total scattering cross section becomes

$$
\sigma_{\chi N}^{\mathrm{NR}}=\frac{g_{d}^{2} \mu_{\chi N}^{2}}{\pi\left(1-\varepsilon^{2}\right)}\left(Z\left(\frac{s_{X} g_{C}}{m_{Z}^{2}}-\frac{c_{X} g_{C d}}{m_{Z_{d}}^{2}}\right)+(A-Z)\left(\frac{s_{X} g_{A}}{m_{Z}^{2}}-\frac{c_{X} g_{A d}}{m_{Z_{d}}^{2}}\right)\right)^{2} .
$$

[1] A. Del Popolo, Int. J. Mod. Phys. D 23, 1430005 (2014).

[2] E. Aprile et al. (XENON Collaboration), Phys. Rev. Lett. 121, 111302 (2018).

[3] R. Agnese et al. (SuperCDMS Collaboration), Phys. Rev. Lett. 121, 051301 (2018).

[4] M. Crisler et al. (SENSEI Collaboration), Phys. Rev. Lett. 121, 061803 (2018).

[5] R. Essig, A. Manalaysay, J. Mardon, P. Sorensen, and T. Volansky, Phys. Rev. Lett. 109, 021301 (2012).

[6] C. V. Cappiello, K. C. Y. Ng, and J. F. Beacom, Phys. Rev. D 99, 063004 (2019).

[7] T. Bringmann and M. Pospelov, Phys. Rev. Lett. 122, 171801 (2019).

[8] Y. Ema, F. Sala, and R. Sato, Phys. Rev. Lett. 122, 181802 (2019).

[9] C. Cappiello and J. F. Beacom, Phys. Rev. D 100, 103011 (2019).

[10] J. B. Dent, B. Dutta, J. L. Newstead, and I. M. Shoemaker, Phys. Rev. D 101, 116007 (2020).

[11] K. Bondarenko, A. Boyarsky, T. Bringmann, M. Hufnagel, K. Schmidt-Hoberg, and A. Sokolenko, J. High Energy Phys. 03 (2020) 118.

[12] G. Guo, Y.L. S. Tsai, and M. R. Wu, arXiv:2004.03161.

[13] W. Wang, L. Wu, J. M. Yang, H. Zhou, and B. Zhu, arXiv: 1912.09904.

[14] L. Su, W. Wang, L. Wu, J. M. Yang, and B. Zhu, arXiv:2006 .11837.

[15] K. Babu, C. F. Kolda, and J. March-Russell, Phys. Rev. D 57, 6788 (1998).

[16] M. Pospelov, A. Ritz, and M. B. Voloshin, Phys. Lett. B 662, 53 (2008).

[17] P. Langacker, Rev. Mod. Phys. 81, 1199 (2009).

[18] R. Foot and S. Vagnozzi, Phys. Rev. D 91, 023512 (2015).

[19] R. Foot and S. Vagnozzi, Phys. Lett. B 748, 61 (2015).

[20] R. Foot and S. Vagnozzi, J. Cosmol. Astropart. Phys. 07 (2016) 013.
[21] T. Emken, R. Essig, C. Kouvaris, and M. Sholapurkar, J. Cosmol. Astropart. Phys. 09 (2019) 070.

[22] M. Ibe, S. Kobayashi, Y. Nakayama, and S. Shirai, J. High Energy Phys. 04 (2020) 009.

[23] K. Y. Choi, K. Kadota, and I. Park, Phys. Lett. B 771, 162 (2017).

[24] E. J. Chun, J. C. Park, and S. Scopel, J. High Energy Phys. 02 (2011) 100.

[25] C. Y. Li, Z. G. Si, and Y. F. Zhou, Nucl. Phys. B945, 114678 (2019).

[26] M. Dutra, M. Lindner, S. Profumo, F. S. Queiroz, W. Rodejohann, and C. Siqueira, J. Cosmol. Astropart. Phys. 03 (2018) 037.

[27] N. Bernal, X. Chu, S. Kulkarni, and J. Pradler, Phys. Rev. D 101, 055044 (2020).

[28] D. Baxter, Y. Kahn, and G. Krnjaic, Phys. Rev. D 101, 076014 (2020).

[29] R. Essig, J. Mardon, and T. Volansky, Phys. Rev. D 85, 076007 (2012).

[30] R. H. Helm, Phys. Rev. 104, 1466 (1956).

[31] D. Bisschoff, M. S. Potgieter, and O.P. M. Aslam, Astrophys. J. 878, 59 (2019).

[32] S. F. Ge, J. L. Liu, Q. Yuan, and N. Zhou, arXiv:2005.09480.

[33] E. Aprile et al. (XENON Collaboration), J. Cosmol. Astropart. Phys. 04 (2016) 027.

[34] C. Kachulis et al. (Super-Kamiokande Collaboration), Phys. Rev. Lett. 120, 221301 (2018).

[35] E. Armengaud et al. (EDELWEISS Collaboration), Phys. Rev. D 99, 082003 (2019).

[36] J. P. Lees et al. (BABAR Collaboration), Phys. Rev. Lett. 113, 201801 (2014).

[37] S. Alekhin et al., Rep. Prog. Phys. 79, 124201 (2016).

[38] G. Krnjaic and S. D. McDermott, Phys. Rev. D 101, 123022 (2020).

[39] H. Baer, K. Y. Choi, J. E. Kim, and L. Roszkowski, Phys. Rep. 555, 1 (2015). 